\title{
Leitlinien für die Diagnostik und Therapie in der Pädiatrischen Onkologie und Hämatologie
}

\author{
Ursula Creutzig, Kurt Winkler und Gïnter Henze
}

\begin{abstract}
Hintergrund Die gesundheitspolitischen Veränderungen nach dem Gesundheitsstrukturgesetz und die Aussichten auf weitere Reformen haben dazu geführt, daß im Dialog zwischen den Ärzten als „Leistungserbringern“" und den Krankenkassen als Kostenträgern gemeinsam neue Konzepte zur Problemlösung entwickelt werden. Ein Weg, um das System durch Selbststeuerung zu stabilisieren, ist die Definition des augenblicklichen Standes des medizinischen Wissens und der ärztlichen Praxis. Fachspezifische Leitlinien können ein Instrument der Qualitätssicherung sein und dem Arzt Sicherheit im ärztlichen Handeln und im juristischen Sinn geben.
\end{abstract}

Derzeit werden Leitlinien von allen medizinischen Fachgesellschaften erarbeitet. Dieses Projekt wird von der Arbeitsgemeinschaft der wissenschaftlichen medizinischen Fachgesellschaften (AWMF) koordiniert. Die Gesellschaft für Pädiatrische Onkologie und Hämatologie ist aufgerufen. Leitlinien bei onkologischen und hämatologischen Erkrankungen im Kindesalter in Abstimmung mit anderen Fachdisziplinen, wie z. B. Kinderchirurgen und Kinderradiologen, und eingebettet in entsprechende Bemühungen der Deutschen Gesellschaft für Kinderheilkunde zu formulieren.

In der Pädiatrischen Onkologie sind sowohl die Diagnostik als auch die Therapie zunehmend komplex geworden. $\mathrm{Zu}-$ dem müssen sie laufend der neuen Entwicklung angepaßt werden. Für den weniger mit dieser Problematik Vertrauten sind manche Maßnahmen u. U. schwer nachvollziehbar. Gerade deshalb ist die Formulierung von Leitlinien erforderlich, die als Konsens von uns a!s Fachvertretern zu verstehen sind und unsere Auffassung über notwendige, sinnvolle und nach dem Stand der Wissenschaft bei einer bestimmten Erkrankung anzuwendende diagnostische und therapeutische Verfahren darstellen.

Definition Standards werden generell als Oberbegriff für Richtlinien, Leitlinien und Empfehlungen verstanden. Hinsichtlich der Verbindlichkeit können Leitlinien zwischen Richtlinien und Empfehlungen eingeordnet werden (Weißauer, AWMF-Protokoll, Oktober 1995). Leitlinien sind Empfehlungen, denen nicht unter allen Umständen gefolgt werden muß. Der Arzt muß die Entscheidung darüber je nach den individuellen Patientengegebenheiten treffen (Clinical Practice Guideline der Agency for Health Care Policy and Research - AHCPR. USA). Es können auch verschiedene Möglichkeiten der Behandlung (Standards) infrage kommen, ohne daß einer Methode oder einer Therapie der Vorzug gegeben werden muß.

Im juristischen Sinn können Leitlinien den Arzt bei der Behandlung des einzelnen Patienten nicht binden. Im

Klin. Pädiatr. 208 (1996) 1.39-140)

(1) 1996 F. Enke Verlag Stuttgart
Zweifelsfall kann aber ein Gericht fragen. warum sich ein Arzt nicht an die formulierten Empfehlungen gehalten hat.

Ziele Leitlinien sind systematisch entwickelte Darstellungen mit dem Ziel, Ärzte und Patienten bei der Entscheidung über zweckdienliche Maßnahmen der Krankenversorgung unter spezifischen klinischen Umständen zu unterstützen.

Die Leitlinie gibt den Stand des Wissens zum Zeitpunkt der Drucklegung wieder. Ein bestimmtes Qualitätsniveau wird beschrieben, das sich am jeweiligen Stand der Wissenschaft (üblicherweise im Lehrbuch publizierte Regeln) und an der klinisch-ärztlichen Praxis orientiert.

Die Entwicklung von Leitlinien muß grundsätzlich durch Experten erfolgen. Die Kompetenz für die Festlegung medizinischer Standards liegt bei den medizinischen Fachvertretern bzw. Fachgesellschaften.

In der Pädiatrischen Onkologie und Hämatologie geht es darum, jedem betroffenen Kind eine adäquate Diagnostik und Therapie zu sichern. Dies entspricht der UNO-Konvention von 1989, nach der jedes Kind (jeder Mensch unter 18 Jahren) Anspruch auf besondere Fürsorge und Unterstützung hat. Die Leitlinien stellen dabei den Konsens für die Angemessenheit der spezifischen ärztlichen Leistungen dar.

Derzeitiger Stand der Entwicklung von Leitlinien Der Vorstand der GPOH fördert die Entwicklung von Leitlinien. Für Kinder mit bösartigen Erkrankungen gibt es im Rahmen unserer Gesellschaft seit etwa 25 Jahren für die meisten Tumoren und Systemerkrankungen des Kindesalters kooperative Studien, die neben einem Behandlungsplan auch diagnostische Empfehlungen enthalten. Durch langjährige Erfahrungen. die im Rahmen solcher Therapieoptimierungsstudien gewonnen werden konnten, sind laufend aktualisierte sogenannte Therapieprotokolle entstanden, die einer optimalen Behandlung der Patienten dienen und gleichzeitig dank der damit hergestellten Einheitlichkeit der Behandlung eine Beurteilung der Behandlungsergebnisse in allen ihren Aspckten ermöglichen.

In die nun ausgearbeiteten Leitlinien fließen grundsätzliche und allgemeingültige Erfahrungen in der Diagnostik und Therapie aus den Studien ein. Es bleibt dabei noch Spielraum für individuelle Entscheidungen. Die grundsätzliche Linie entspricht jedoch dem Konsens der Experten, wie er nach erneuter Diskussion mit den verschiedenen beteiligten Fachgruppen erarbeitet wurde. Dies bedeutet, daß grobe Abweichungen in Diagnostik oder Therapie begründbar sein müssen. Die Einschränkung des eigenen Ermessensspielraumes wird reichlich aufgewogen durch eine Sicherheit für unsere schweren Entscheidungen im Altag. Der in den Leitlinien formulierte gemeinsame 
Standpunkt bekräftigt darüber hinaus auch unsere Verhandlungsposition gegenüber Gesundheitspolitikern, Krankenkassen und Krankenhausadministration bei der Feststellung und Durchsetzung dessen, was notwendig und zureichend ist für die Behandlung der uns anvertrauten Kinder.

Die Leitlinien für die pädiatrisch-onkologischen Krankheitsbilder werden in Kürze im Rahmen der Reihe Qualitätssicherung in der Onkologie von der Deutschen Krebs- gesellschaft herausgegeben. Es folgt eine weitere Publikation durch die Deutsche Gesellschaft für Kinderheilkunde, die auch die nicht-onkologischen hämatologischen Diagnosen enthalten wird.

Priv.-Doz. Dr. Ursula Creutzig

Univ.-Kinderklinik

Albert-Schweitzer-Str. 33

D-48129 Münster 